\title{
Acupuncture for the Treatment of Hot Flashes in Men with Advanced Prostate Cancer
}

\author{
Jillian L. Capodice, Philippa Cheetham, Mitchell C. Benson, James M. McKiernan, Aaron E. Katz
}

Department of Urology, Columbia University Medical Center, New York, USA.

Email: \{jc2346, mcb2,jmm23, aek4\}@columbia.edu, pcheetham@doctors.ork.uk

Received December $14^{\text {th }}, 2010$; revised January $6^{\text {th }}, 2011$; accepted January $11^{\text {th }}, 2011$.

\begin{abstract}
PURPOSE: To test the safety and efficacy of acupuncture in reducing hot flashes in men with advanced prostate cancer $(\mathrm{CaP})$ undergoing androgen deprivation therapy. METHODS: A single-arm, pilot study was undertaken to evaluate acupuncture treatment for hot flashes experienced by CaP patients undergoing hormonal therapy. Sixteen advanced $C a P$ patients undergoing androgen deprivation therapy $(A D T)$ received standardized full body and auricular acupuncture $1 x /$ week for 14 weeks. Patients were evaluated at $0,7,14$, and an additional 14-wk follow-up (F/U) (28-wks). Safety was measured by monitoring for adverse events over the treatment period. Serum testosterone was measured at 0 and 14-wks. Quality of life (QOL) was measured by the hormone domain of the Expanded Prostate Index Composite (EPIC), at the 0, 7, 14, and 28-wks and patient reported hot flash frequency was assessed weekly. Safety endpoints were serum testosterone and analysis of adverse events. Efficacy endpoints were scores on the EPIC and the number of patient reported hot flashes. RESULTS: 17 patients were enrolled and 15 completed the trial. Of 15 evaluable patients the median age was $68 \pm 8.19$. Serum analysis demonstrated no change in testosterone at baseline $9.5 \mathrm{ng} / \mathrm{ml} \pm 8.9 \mathrm{vs}$. endpoint $14 \pm 8.78(p=0.101)$. No adverse events were reported. Data analysis of the EPIC demonstrated a trend toward improvement after 7 wks and a significant improvement following 14 wks of acupuncture $(p=0.01)$. Analysis of patient reported frequency showed a significant reduction in the number of hot flashes at 7-wk (6, $p=0.04), 14-w k(2.6, p=$ $0.001)$, and 28-wk F/U (3.2, $p=0.01)$ as compared to baseline $9.57 \pm 3.98$. CONCLUSIONS: The administration of acupuncture in men with advanced CaP appears to significantly decrease the frequency of hot flashes. No serious adverse events were noted and serum testosterone levels were unchanged from baseline suggesting that the mechanism of action of acupuncture for hot flash amelioration is not via increase in testosterone.
\end{abstract}

Keywords: Prostate Cancer, Acupuncture, Hot Flashes

\section{Background}

Prostate cancer $(\mathrm{CaP})$ is the most commonly diagnosed cancer in American men and the second leading cause of cancer deaths. More than 217,730 men will be diagnosed with prostate cancer in 2010, and an estimated 32,050 patients will die of this disease [1,2]. Factors including family history and race/ethnicity account for increased risk of prostate cancer. Age-specific incidence rates are also a factor in $\mathrm{CaP}$, and advanced age accounts for about $60 \%$ all newly diagnosed prostate cancer cases. Additionally, $80 \%$ of all $\mathrm{CaP}$ deaths occur in men greater than 70 years of age [2].

However, prostate cancer $(\mathrm{CaP})$ patients are living longer largely due to the benefits of androgen deprivation therapy (ADT) including androgen ablation utilizing luteinizing hormone-releasing hormone (LHRH) agonists alone or in combination with anti-androgens. This treatment has become a standard option for men with metastatic CaP (Stage 3 or 4) but frequently cause multiple side effects including gyencomastia, fatigue, hair loss and hot flashes. The cause of hot flashes is thought to be due to the decrease in circulating leutinzing hormone (LH) and follicle stimulating hormone (FSH) [3-5] and the incidence of hot flashes in men with $\mathrm{CaP}$ is close to $80 \%$ over the course of treatment and often continues for years even after ADT is stopped [3]. The severity of hot flashes impact quality of life and often interfere with patient compliance. The most commonly prescribed medication to ameliorate hot flashes are estrogens which also cause undesirable side effects [4] and in the management of patients with advance cancers, maintaining treatment compliance, minimizing side effects and preserving quality of life (QOL) are of extreme importance 
[3,4]. Based on these facts we hypothesize that acupuncture, a popular complementary and alternative medicine (CAM) modality may offer a non-pharmacologic approach to the amelioration of hot flashes in men with advanced prostate cancer.

\section{Materials and Methods}

\subsection{Subjects}

Men with a history of prostate cancer (CaP) Stage 3 (T3, N0, M0, any G) or Stage 4 (T4, N0, M0, any G or any T, $\mathrm{N} 1, \mathrm{M} 0$ any G), currently taking any hormonal therapies including leuprolide acetate or goserelin acetate ( \pm flutamide, nilutamide, or bicalutamide), reporting four or more hot flashes/day, able to walk, able to lie prone for the duration of treatment and able to sign the informed consent in English were eligible for the study. Excluded from the study were men with the presence of any bleeding disorder or skin infection, any acupuncture treatment within the past 6 months, initiation or cessation of other hormonal therapy during or for the 3 weeks prior to the start of the study, current use or introduction of any pharmacological treatment for hot flashes, current chemotherapy or chemotherapy within the past 3 months, current radiation or completed radiation within the past month, and current use of any of the following dietary supplements including non-dietary soy, Cimicifuga racemosa (black cohosh), Trifolium pretense (red clover), or vitamin E (> 800 IU daily). The protocol for this pilot study was approved by the Institutional Review Board of Columbia University Medical Center and the Herbert Irving Comprehensive Cancer Center (New York, NY, USA).

\subsection{Study Procedures}

Consecutive subjects were assessed at their initial enrollment and if they met the eligibility criteria were instructed to read, understand and sign the written informed consent Subjects were then assigned to onceweekly 30-minute acupuncture sessions for 14 weeks. At baseline subjects were asked to complete demographic information. During each weekly visit, the subject was asked to report their average number of hot flashes per day. At baseline (week 0), week 7 and 14, blood draws were taken. At weeks $0,7,14$, and 28 the EPIC questionnaire was administered.

\subsection{Acupuncture Intervention}

The acupuncture treatment protocol and procedure were devised according to Standard for Reporting of Controlled Trials in Acupuncture (STRICTA) [6]. Our acupuncture rationale included standardized body and auricular treatment and the protocol consisted of a standar- dized set of acupuncture pointes given weekly for 30 minutes over the 14 week period. Points were selected based on standard texts, informal practitioner query, and previous studies on acupuncture for hot flashes [7-9] and all points were applied to standard needle depths. All acupuncture points were administered with the patient lying in the prone position, with feet elevated by a pillow. Stainless steel acupuncture needles were single-use, sterile and disposable. Full body acupuncture needles were 32 and 34 gauge (Cloud and Dragon, Wujiang City Cloud and Dragon Medical Device Co. Ltd, China). Auricular acupuncture needles were $15 \mathrm{~mm}$ and 38 gauge (Seirin, Seirin-America Inc., Weymouth, MA). All needles were inserted and a de qi sensation was obtained at each point [10]. The needles remained in situ for 30 minutes during which time the acupuncturist returned to stimulate the needles once (light rotation of the needle back and forth up to six times). The full body acupuncture point prescription was: SP 6, UB 15, UB 23, UB 43, LR 3, KD 6, LU 7, HT 7. The Auricular Acupuncture Point Prescription was: Shen Men, Brain, Kidney, Liver, and Upper Lung. All treatments were provided by a single acupuncturist licensed in New York State, USA throughout the study period.

\subsection{Statistics}

The primary safety outcome was the incidence of adverse effects, complication/illness and/or serious medical events most likely due to the acupuncture treatment. Other safety measurements included measurement serum testosterone levels. The primary efficacy outcome of this study was change in the score of the hormone domain of the EPIC questionnaire. Secondary outcomes were reduction in self-reported hot flashes and hot flash frequency based on self-reported daily diaries. The acupuncture treatment effect was assessed by a paired t- test comparing measurements of the symptom survey at baseline to weeks 7, 14 and 28 for all applicable measures. Descriptive analyses were performed for demographics utilizing characteristic measures such as mean, standard deviation, range etc. Analyses were performed using SPSS Version 9.0 for Windows (Chicago, IL).

\section{Results}

\subsection{Subjects}

Seventeen men with advanced prostate cancer were enrolled in the trial. The mean \pm SD age was $68.06 \pm$ 8.19. Demographics and baseline characteristics are summarized in Table 1. Eighty-eight point two percent of the men (15/17) completed the trial. The reasons for premature withdrawal were presence of a second cancer [1] and moving [10]. 
Table 1. Demographics.

\begin{tabular}{cc}
\hline Characteristic & Frequency \\
\hline N & 17 \\
Median age, years \pm SD & $68.06 \pm 8.19$ \\
Race, no (\%) & $10(58.8)$ \\
White & $5(29.4)$ \\
Black & $1(5.9)$ \\
Hispanic/Latino & $1(5.9)$ \\
Asian & \\
LHRH agonist (\%) & $17 / 17(100)$ \\
Anti-androgen (\%) & $11 / 17[64.7]$ \\
Baseline hot flash frequency & $9.57 \pm 3.98$ \\
\hline
\end{tabular}

\subsection{Safety and Tolerability Outcomes}

No serious side effects were reported throughout treatment and follow-up including no reports of bleeding, bruising at any needle site, dizziness or vasovagal response(s) that may have been directly related to administration of full body or auricular acupuncture treatment. Testosterone levels at 0 and 14 weeks showed no change in testosterone at baseline $9.5 \mathrm{ng} / \mathrm{ml} \pm 8.9 \mathrm{ng} / \mathrm{ml}$ from the endpoint average of $14.0 \pm 8.78, \mathrm{p}=0.101$ (Table 2).

\subsection{EPIC Data and Hot Flash Frequency}

\subsubsection{EPIC}

Analysis of the EPIC questionnaire (hormonal domain) demonstrated a trend toward improvement following 7 weeks of treatment and a significant improvement following 14 weeks of acupuncture $(p=0.006)$. However after an additional 14 weeks of follow up the trend was reduced $(\mathrm{p}=0.055)$, see Figure 1 .

\subsubsection{Hot Flash Frequency}

At the 7-wk follow-up patients reported a significant decrease in hot flashes $(\mathrm{p}=0.04)$ with an average of 6.00 \pm 2.4 hot flashes per day as compared to $9.57 \pm 3.98$ at baseline. The trend in decreasing frequency of hot flashes continued at the 14-wk follow-up, with patients reporting an average of $2.63 \pm 1.2$ hot flashes per day. The 14-wk numbers represent a significant improvement from the baseline frequency of hot flashes $(\mathrm{p}=0.001)$ (Table 2). The decrease in hot flush frequency was maintained over the following 14 weeks without acupuncture treatments, and at the 28-wk follow-up while the value was slightly increased, the subjects still reported decreased hot flash frequency as compared to baseline $3.2 \pm 1.7(\mathrm{p}=0.01)$.

\section{Discussion}

The results of our study demonstrate that acupuncture was safe, well tolerated, and significantly reduced the frequency and severity of hot flashes experienced by men

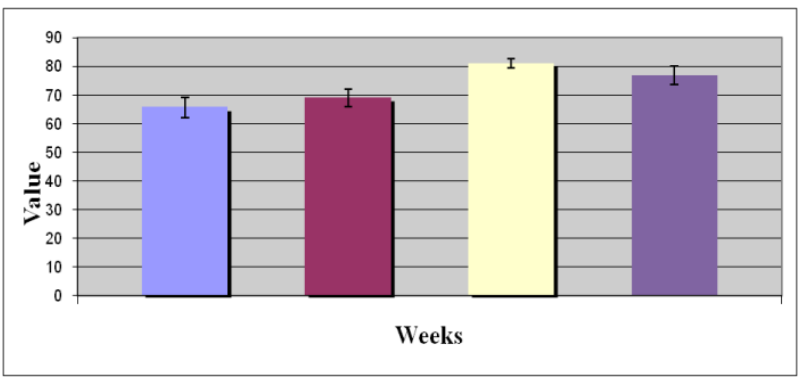

Figure 1. Results of the EPIC-hormone domain *. Student's t-test week 0 versus weeks 7,14 and $28(p=0.028, p=0.006$, $\mathbf{p}=\mathbf{0 . 0 5 5}$ respectively), * Higher values represent a better outcome, $* * \mathbf{p}<0.05$.

Table 2. Change in weekly reported hot flashes and testosterone.

\begin{tabular}{cccc}
\hline Description & $\begin{array}{c}\text { Baseline } \\
\text { mean (SD) }\end{array}$ & $\begin{array}{c}\text { After acu- } \\
\text { puncture, week } \\
14 \text { (SD) }\end{array}$ & $P$-value \\
\hline $\begin{array}{c}\text { Self-reported } \\
\text { hot flashes } \\
\begin{array}{c}\text { Testosterone } \\
\text { (ng/ml) }\end{array}\end{array}$ & $9.57 \pm 3.98$ & $2.63 \pm 1.2$ & $\mathrm{P}=0.001$ \\
\hline
\end{tabular}

undergoing hormonal therapy for advanced prostate cancer as measured by a standardized questionnaires and other self reported outcomes. Moreover, the administration of acupuncture did not affect testosterone levels throughout the study period, an additional safety benchmark for patients being treated by androgen ablation.

Our results demonstrate that acupuncture significantly affected scores on the hormonal portion of the EPIC questionnaire after 14 weeks of treatment $(p=0.10)$ but only trended toward improvement at 7 weeks $(p=0.288)$. This suggests that the treatment dosage with regard to application of acupuncture for hot flashes may be longer than dosages of acupuncture for other conditions. For example, studies of acupuncture for post-operative or chronic osteoarthritic pain demonstrate that amelioration of pain (measured by standardized questionnaires) may occur as quickly as 2-3 weeks [11]. Our second measure of weekly patient reported hot flash frequency was also significant at weeks 7, 14 and 28 and these data were consistent with previous reports [12].

After the 14 week study period, serum testosterone remained unchanged despite significant amelioration of hot flashes by full body and auricular acupuncture. This finding is extremely important as it demonstrates that acupuncture is safe when administered to patients receiving hormonal therapy and that the potential mechanism of action is not via testosterone increase, an outcome which would be contraindicated in this patient population. Interestingly the most recent data analyzing the effect of acupuncture for hot flashes in both men with prostate 
cancer and women with breast cancer imply a neuromodulatory mechanism via neurotransmitter release [13-15]. This hypothesis is also supported by recent clinical trials on selective serotonin reuptake inhibitors for the treatment for hot flashes in both men and women $[14,15]$ and recent in vitro data demonstrating that acupuncture may increase circulating serotonin in a murine model [16].

A handful of other studies of acupuncture for hot flashes induced by hormonal therapy in men also suggest a potential neuromodulatory mechanism of action including b-endorphin release and potential effect on calcitonin gene-related peptide immunoreactivity $[7,13]$. Based on our original hypothesis and current research, we also suggest that the action of acupuncture in men with hot flashes may be due to a neuromodulatory effect that targets noradrenergic and serotonergic pathways that are thought to be involved with hypothalamic thermoregulation following the reduction of testosterone and hormonal fluctuations as a result of androgen ablation. Moreover, fMRI research has recently implied that acupuncture modulates cortical activity and may powerfully modulate human subjective experiences including responses to pain, hunger, and memory [17].

Limitations of our study include lack of a placebo or sham control. However at the time of study design only one case report existed in the literature and we felt that pilot data needed to be collected first in order to support a hypothesis for a larger trial.

Our study clearly demonstrates that acupuncture is safe and effective in reducing hot flashes in men with advanced prostate cancer undergoing androgen deprivation therapy following 14 weeks of acupuncture treatment. It is warranted for further controlled studies that should investigate the potential mechanism of action of acupuncture and may be a powerful treatment for a debilitating symptom in which there is limited medical armamentarium.

\section{REFERENCES}

[1] A. Jemal, R. Siegel, J. Xu and E. Ward, "Cancer Statistics,” CA: A Cancer Journal of Clinician, Vol. 60, No. 5, September-October 2010, pp. 277-300.

[2] M. B. Garnick, "Prostate Cancer: Screening, Diagnosis, and Management," Annals of Internal Medicine, Vol. 118, No. 10, 1993, pp. 804-818.

[3] C. S. Higano, "Side Effects of Androgen Deprivation Therapy: Monitoring and Minimizing Toxicity,” Urology, Vol. 61, No. 2, Suppl. 1, 2003, pp. 32-38. doi:10.1016/S0090-4295(02)02397-X

[4] J. Anderson, "The Role of Antiandrogen Monotherapy in the Treatment of Prostate Cancer," British Journal of Urology International, Vol. 91, No. 5, 2003, pp. 455-461.

[5] K. Suzuki, M. Kobayashi and A. Tokue, "Clinical Evalu- ation of Hot Flushes Developing during Endocrine Therapy for Prostate Carcinoma,” Nippon Hinyokika Gakkai Zasshi, Vol. 94, No. 6, 2003, pp. 614-620.

[6] H. MacPherson, A. White, M. Cummings, K. Jobst, K. Rose and R. Niemtzow, "Standards for Reporting Interventions in Controlled Trials of Acupuncture: The STRICTA Recommendations," Complementary Therapies in Medicine, Vol. 9, No. 4, 2001, pp. 246-249. doi:10.1054/ctim.2001.0488

[7] M. Hammar, J. Frisk, O. Grimas, et al., "Acupuncture Treatment of Vasomotor Symptoms in Men with Prostatic Carcinoma: A Pilot Study,” Journal of Urology, Vol. 161, No. 3, 1999, pp. 853-856.

doi:10.1016/S0022-5347(01)61789-0

[8] X. Chen, "Chinese Acupuncture and Moxibustion," Foreign Languages Press, Beijing, 1999.

[9] Y. Wu and W. Fisher, "Practical Therapeutics of Traditional Chinese Medicine,” Paradigm Publications, Brookline, 1997.

[10] A. U. Asghar, G. Green, M. F. Lythgoe, G. Lewith and H. MacPherson, "Acupuncture Needling Sensation: The Neural Correlates of DEQI Using fMRI,” Brain Research, Vol. 22, No. 1315, 2010, pp. 111-118. doi:10.1016/j.brainres.2009.12.019

[11] E. Manheimer, K. Linde, L. Lao, L. M. Bouter and B. M. Berman, "Meta-Analysis: Acupuncture for Osteoarthritis of the Knee," Annals of Internal Medicine, Vol. 146, No. 12, 2007, pp. 868-867.

[12] L. J. Hanisch, S. C. Palmer, S. C. Marcus, L. Hantsoo, D. J. Vaughn and J. C. Coyne, "Comparison of Objective and Patient-Reported Hot Flash Measures in Men with Prostate Cancer," Journal of Supportive Oncology, Vol. 7, No. 4, 2009, pp. 131-135.

[13] C. Harding, A. Harris and D. Chadwick, "Auricular Acupuncture: A Novel Treatment for Vasomotor Symptoms Associated with Luteinizing-Hormone Releasing Hormone agonist Treatment for Prostate Cancer," British Journal of Urology International, Vol. 103, No. 2, 2009, pp. 186-190.

[14] C. L. Loprinzi, A. C. Dueck, B. S. Khoyratty, D. L. Barton, S. Jafar, K. M. Rowland Jr, P. J. Atherton, G. W. Marsa, W. H. Knutson, J. D. Bearden 3rd, L. Kottschade and T. R. Fitch, "A Phase III Randomized, Double-Blind, Placebo-Controlled Trial of Gabapentin in the Management of Hot Flashes in Men (N00CB)," Annals of Oncology, Vol. 20, No. 3, 2009, pp. 542-549. doi:10.1093/annonc/mdn644

[15] E. M. Walker, A. I. Rodriguez, B. Kohn, R. M. Ball, J. Pegg, J. R. Pocock, R. Nunez, E. Peterson, S. Jakary and R. A. Levine, "Acupuncture versus Venlafaxine for the Management of Vasomotor Symptoms in Patients with Hormone Receptor-Positive Breast Cancer: A Randomized Controlled Trial,” Journal of Clinical Oncology, Vol. 28, No. 4, 2010, pp. 634-640. doi:10.1200/JCO.2009.23.5150

[16] A. Moazzami, S. C. Tjen-A-Looi, Z. L. Guo and J. C. Longhurst, "Serotonergic Projection from Nucleus Raphe 
Pallidus to Rostral Ventrolateral Medulla Modulates Cardiovascular Reflex Responses during Acupuncture,” Journal of Applied Physiology, Vol. 108, No. 5, 2010, pp. 1336-1346.

[17] L. Bai, H. Yan, Li L, W. Qin, P. Chen, P. Liu, Q. Gong,
Y. Liu and J. Tian, "Neural Specificity Of Acupuncture Stimulation at Pericardium 6: Evidence from an FMRI Study,” Journal of Magnetic Resonance Imaging, Vol. 31, No. 1, 2010, pp. 71-77. doi:10.1002/jmri.22006 